\title{
Measurement of multiplicities of charged hadrons, pions and kaons in DIS at COMPASS
}

\author{
Nikolai Mitrofanov (On Behalf of COMPASS Collaboration) $)^{1, \star}$ \\ ${ }^{1}$ Joint Institute for Nuclear Research, Dubna
}

\begin{abstract}
Precise measurements of multiplicities of charged hadrons, pions and kaons in deep inelastic scattering were performed. The data were obtained by the COMPASS Collaboration by scattering $160 \mathrm{GeV}$ muons off an isoscalar ${ }^{6} \mathrm{LiD}$ target. The results were obtained in three-dimensional bins of the Bjorken scaling variable $x$, the relative virtual-photon energy $y$, and the fraction $z$ of the virtual-photon energy carried by the produced hadron. A leading-order $\mathrm{pQCD}$ analysis was performed using the pion multiplicity results to extract quark fragmentation functions into pions. The results for the sum of the $z$-integrated multiplicities for pions and for kaons, differ from earlier results from the HERMES experiment. The results from the sum of the $z$-integrated $K^{+}$and $K^{-}$ multiplicities at high $x$ point to a value of the non-strange quark fragmentation function larger than obtained by the earlier DSS fit.
\end{abstract}

\section{Introduction}

Hadron production in semi-inclusive measurements of deep-inelastic lepton-nucleon scattering (SIDIS) is one of the most powerful tools to investigate the structure and formation of hadrons. Within the standard framework of leading-twist perturbative QCD (pQCD), factorisation theorems [3] allow one to write the SIDIS cross section as a convolution of hard scattering cross sections, which are calculable in pQCD, with non-perturbative Parton Distribution Functions (PDFs) and Fragmentation Functions (FFs). The PDFs account for the partonic structure of hadrons in the initial state. The FFs encode the details about the hadronisation mechanism that describes the transition from final-state partons into colour-neutral hadrons. Both types of functions are believed to be process independent, and can be interpreted in leading-order (LO) as number densities. While PDFs have been studied in detail for several decades and are hence known with good precision, new accurate measurements are necessary to constrain the FFs.

The DIS process $l N \rightarrow l^{\prime} h X$ is described by the negative square of the four-momentum transfer $Q^{2}=-q^{2}$, the Bjorken variable $x=-q^{2} /(2 P \cdot q)$ and the fraction of the virtual-photon energy that is carried by the final-state hadron, $z=\left(P \cdot p_{h}\right) /(P \cdot q)$. Here, $l$ and $l^{\prime}$ are beam and scattered leptons respectively, $q=k-k^{\prime}, P$ and $p_{h}$ denote the four-momenta of the virtual photon, the nucleon $N$ and the observed hadron $h$ respectively, with $k\left(k^{\prime}\right)$ the four momentum of the incident (scattered) lepton. Additional variables used are the lepton energy fraction carried by the virtual photon, $y=(P \cdot q) /(P \cdot k)$, and the invariant mass of the final hadronic system, $W=\sqrt{(P+q)^{2}}$. hadronisation mechanism in

\footnotetext{
^e-mail: nikolai.mitrofanov@cern.ch
} 
SIDIS, the relevant observable is the differential multiplicity for hadrons of a specific type $h$, which is defined as the differential cross section for hadron production normalised to the differential inclusive DIS cross section:

$$
\frac{d M^{h}\left(x, z, Q^{2}\right.}{d z}=\frac{d^{3} \sigma^{h}\left(x, z, Q^{2}\right) / d x d Q^{2} d z}{d^{2} \sigma^{D I S}\left(x, Q^{2}\right) / d x d Q^{2}} .
$$

Interpreted in $\mathrm{pQCD}$, the cross sections on the right-hand side are expressed in terms of PDFs and FFs and read at leading-order (LO)

$$
\begin{aligned}
\frac{d^{2} \sigma^{D I S}}{d x Q^{2}} & =C\left(x, Q^{2}\right) \sum_{q} e_{q}^{2} q\left(x, Q^{2}\right), \\
\frac{d^{2} \sigma^{h}}{d x Q^{2} d z} & =C\left(x, Q^{2}\right) \sum_{q} e_{q}^{2} q\left(x, Q^{2}\right) D_{q}^{h}\left(z, Q^{2}\right) .
\end{aligned}
$$

Here, $C\left(x, Q^{2}\right)=2 \pi \alpha^{2}\left(1+(1-y)^{2} / Q^{4}\right.$ with $\alpha$ the fine structure constant, $q\left(x, Q^{2}\right)$ is the quark PDF for the flavour $q$ and $D_{q}^{h}\left(z, Q^{2}\right)$ the quark-to-hadron FF. In LO, $D_{q}^{h}$ denotes the number density of hadrons $h$ produced in the hadronisation of partons of species $q$.

\section{COMPASS experiment}

COMPASS [4] is a fixed-target experiment at a secondary beam of the Super Proton Synchrotron at CERN. The purpose of this experiment is the study of hadron structure and hadron spectroscopy with muon and hadron beams of high intensity. The experiment has an intensive physics programme which includes topics such as a study of nucleon spin structure in the semi-inclusive deep inelastic scattering and Drell-Yan process, search for new exotic hadron states in diffractive, central, Primakoff and leptoproduction, tests of the chiral theory predictions, measurement of generalized parton distributions (GPDs), etc.

The measurement was performed in 2006 with the naturally polarised muon beam of the CERN SPS using positive muons of $160 \mathrm{GeV} / \mathrm{c}$. The beam momentum had a spread of $5 \%$. The intensity was $4 \times 10^{7} \mathrm{~s}^{-1}$ with spills of $4.8 \mathrm{~s}$ and a cycle time of $16.8 \mathrm{~s}$. The momentum of each incoming muon was measured before the COMPASS experiment with a precision of $0.3 \%$. The target consisted of three cells, which were located along the beam one after the other and filled with ${ }^{6} \mathrm{LiD}$ immersed in a liquid ${ }^{3} \mathrm{He} /{ }^{4} \mathrm{He}$ mixture. The admixtures of $\mathrm{H},{ }^{3} \mathrm{He}$ and ${ }^{7} \mathrm{Li}$ in the isoscalar target lead to an effective excess of neutrons of about $0.2 \%$. The direction of the polarisation in the $60 \mathrm{~cm}$ long middle cell was opposite to that in the two $30 \mathrm{~cm}$ long outer cells and was reversed once per day. In the analysis, the data are averaged over the target polarisation for the determination of multiplicities.

\section{Data Analysis}

Differential multiplicities are determined in 3-dimensional $(x, y, z)$ bins from the acceptance-corrected hadron yields $N^{h}$ normalised by the number of DIS events,

$$
\frac{d M^{h}(x, y, z)}{d z}=\frac{1}{N^{D I S}(x, y)} \frac{d N^{h}(x, y, z)}{d z} \frac{1}{A(x, y, z)} .
$$

The acceptance correction factor $A$ takes into account the limited geometric and kinematic acceptance of the spectrometer and the efficiency of event reconstruction. The choice of the $z$ and $x$ variables is natural because multiplicities depend mostly on these variables. Because of the strong correlation between $x$ and $Q^{2}$ in the COMPASS fixed-target kinematics, it appears more appropriate to use y instead of $Q^{2}$ as the third variable. 

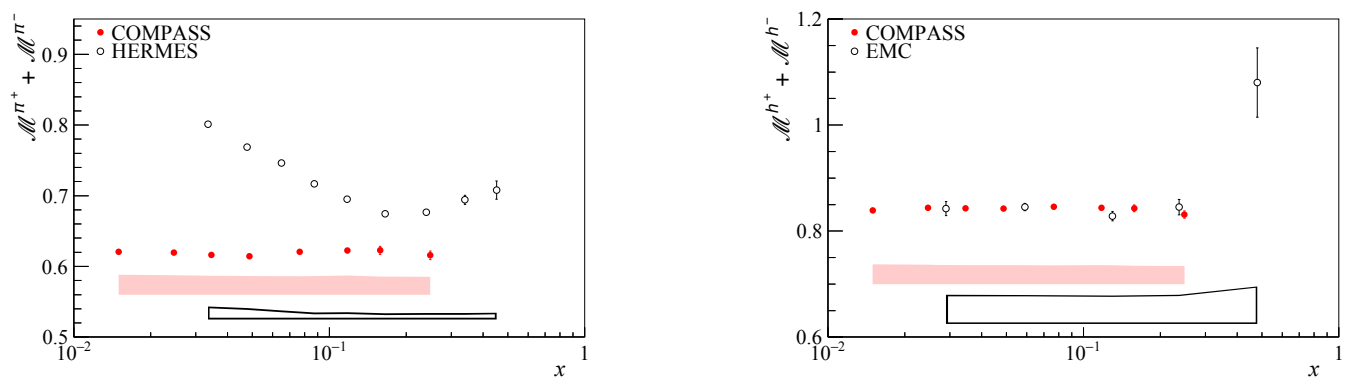

Figure 1. Left: Sum of $\mathscr{M}^{\pi^{+}}+\mathscr{M}^{\pi^{-}}$versus $x$. The COMPASS data (160 GeV, closed circles) are compared to HERMES results (27.5 GeV, open circles); Right: Sum of $\mathscr{M}^{h^{+}}+\mathscr{M}^{h^{-}}$versus $x$. The COMPASS data (160 GeV, closed circles) are compared to EMC results ( $100 \mathrm{GeV}$ to $280 \mathrm{GeV}$, open circles). The systematic uncertainties are shown as bands at the bottom.

\section{Results for multiplicities}

\subsection{Charged pion and charged hadron multiplicities}

On the Fig. 1 (left) is shown the result [1] for the sum $\mathscr{M}^{\pi^{+}}+\mathscr{M}^{\pi^{-}}$of $\pi^{+}$and $\pi^{-}$multiplicities, integrated over $z$ from 0.2 to 0.85 and averaged over $y$ between 0.1 and 0.7 , as a function of $x$. The expected weak $x$ dependence is indeed observed in the data. In the same figure, the results of HERMES [5] integrated over $z$ from 0.2 to 0.8 are shown using the so-called $x$ representation. The HERMES multiplicities are larger and show a different dependence on $x$. Note however that the HERMES data were measured at a lower energy and correspond to different kinematics, in particular accepting lower $W$ values. In order to compare the COMPASS results also with the EMC ones [6], the sum of charged hadron multiplicities is shown in Fig. 1 (right). The results from COMPASS and EMC, which correspond to comparable kinematics, are found in excellent agreement.

Another quantity of interest is the $x$ dependence of the ratio $\mathscr{M}^{\pi^{+}} / \mathscr{M}^{\pi^{-}}$. The results are shown in Fig. 2 (left) as a function of $x$. They are in reasonable agreement with the HERMES values in the measured range. The values obtained from the JLab E00-108 [7] experiment. for $z>0.3$ at higher $x$ and lower $W$ values are also shown for completeness. In Fig. 2 (right), the ratio $\mathscr{M}^{h^{+}} / \mathscr{M}^{h^{-}}$ calculated for charged hadron multiplicities is shown for COMPASS and EMC data. These results are in excellent agreement.

Direct extraction of FFs from pion multiplicities was done [1]. The results from this direct extraction of FFs are in good agreement with the results obtained from the LO fit. This is illustrated in Fig. 3 for one $(x, y)$ bin.

\subsection{Charged kaon multiplicities}

The result [2] for $\mathscr{M}^{K^{+}}+\mathscr{M}^{K^{-}}$is presented in Fig.4 (left) as a function of $\mathrm{x}$ at the measured values of $Q^{2}$. The data are integrated over $z$ in the range 0.20 to 0.85 and averaged over $y$ in the range 0.1 to 0.7 . Only those eight $x$ bins which have a sufficient $z$ coverage are shown. Fig. 4 (left) also shows the HERMES results [8] that were taken at $27.5 \mathrm{GeV}$ beam energy and correspond to different kinematics in particular accepting lower $W$ values. They lie well below the COMPASS points and exhibit a different $x$ behaviour. The $x$ dependence of the HERMES results for pion and kaon multiplicities gave rise to some dispute [9-11]. Note that COMPASS multiplicities are computed in bins of ( $x, y$, 

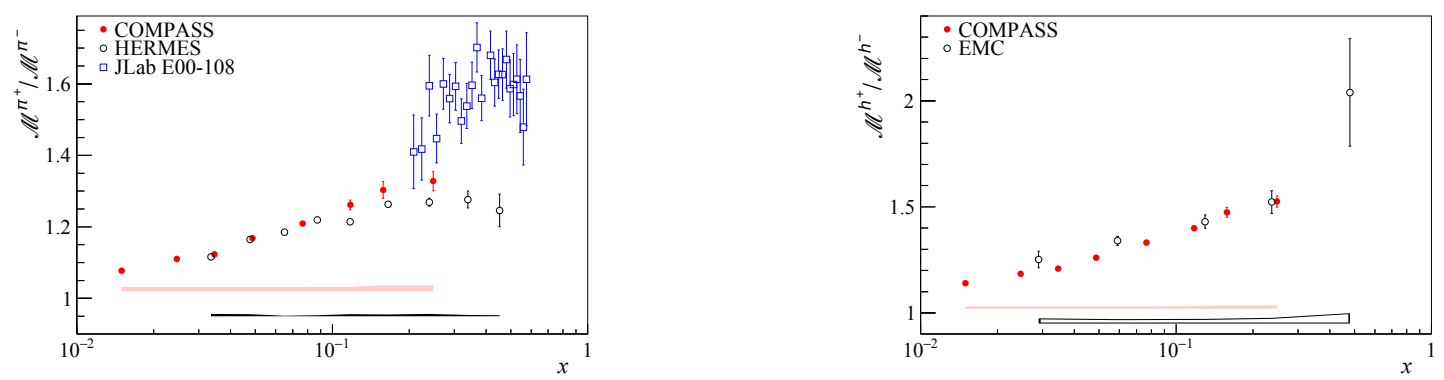

Figure 2. Left: Ratio $\mathscr{M}^{\pi^{+}} / \mathscr{M}^{\pi^{-}}$versus $x$ from COMPASS (160 GeV, closed points), HERMES (27.5 GeV, open circles) and JLab (5.5 GeV, open squares). Right: Ratio $\mathscr{M}^{h^{+}} / \mathscr{M}^{h^{-}}$versus $x$ for COMPASS $(160 \mathrm{GeV}$, closed circles) and EMC ( $100 \mathrm{GeV}$ to $280 \mathrm{GeV}$, open circles) results. The systematic uncertainties are shown as bands at the bottom.

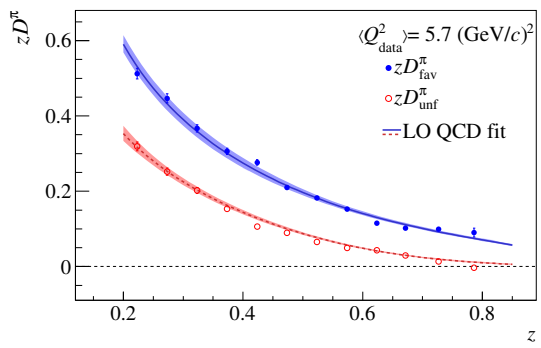

Figure 3. The $z$ dependence of the favoured (closed symbols) and unfavoured (open symbols) quark-to-pion fragmentation functions, $z D_{f a v}^{\pi}$ and $z D_{u n f}^{\pi}$, extracted directly from pion multiplicities at the measured $Q^{2}$ for one $(x, y)$ bin $(0.04<x<0.06,0.3<y<0.5)$. For comparison, the result from the present LO QCD fit to the pion multiplicities is shown at $Q^{2}=6(\mathrm{GeV} / \mathrm{c})^{2}$. The bands represent the total uncertainties of the QCD fit.

$z$ ) before integration over $z$ and averaging over $y$ while in the case of HERMES, the hadron and DIS yields are obtained and integrated over separately, and finally combined in a ratio depending on $x$ only [11].

The results for the ratio are shown in Fig. 4 (right) as a function of $x$. In the region of overlap, COMPASS results are found to be systematically lower than those of HERMES.

\section{Summary and conclusions}

Precise results for the multiplicities of charged-kaons, charged-pions and hadrons are obtained from SIDIS measurements using a muon beam scattering off an isoscalar target. The data are given in a three-dimensional $x, y$, and $z$ binning and cover a wide kinematic range: $1(\mathrm{GeV} / \mathrm{c})^{2}<Q^{2}<60$ $(\mathrm{GeV} / \mathrm{c})^{2}, 10^{-3}<x<0.4,0.1<y<0.7$, and $0.20<z<0.85$ with $\mathrm{W}>5 \mathrm{GeV} / \mathrm{c}^{2}$. These high precision multi-dimensional data provide an important input for future NLO QCD fits of fragmentation functions. 

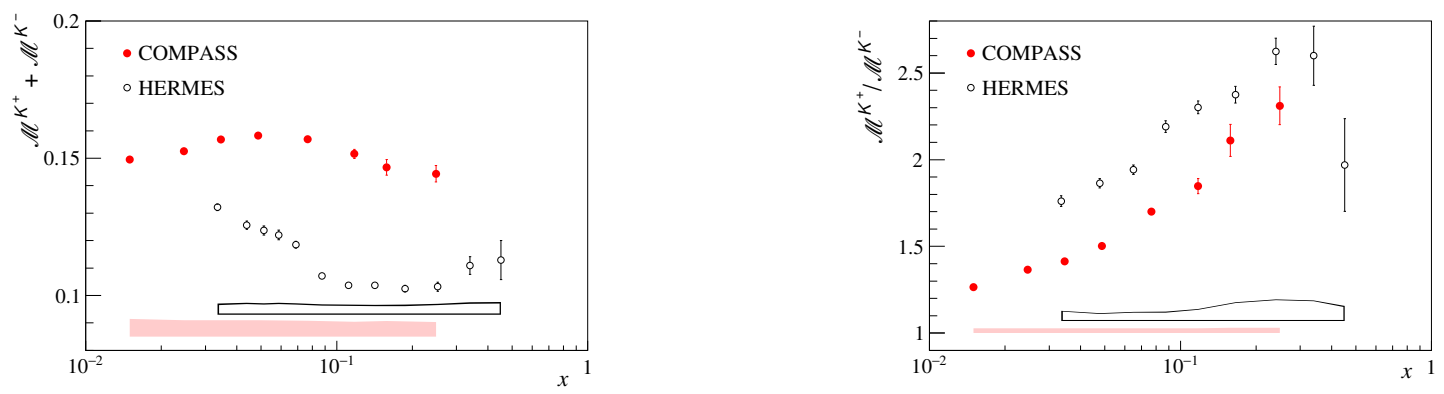

Figure 4. Left: sum of $z$-integrated multiplicities, $\mathscr{M}^{K^{+}}+\mathscr{M}^{K^{-}}$. COMPASS data (160 GeV, full points) are compared to HERMES data [8] (27.5 GeV, open points). Right: Ratio of $z$-integrated multiplicities, $\mathscr{M}^{K^{+}} / \mathscr{M}^{K^{-}}$. COMPASS data (160 GeV, full points) are compared to HERMES data [12] (27.5 GeV, open points). The systematic uncertainties are shown as bands at the bottom.

The sum of the $z$-integrated positively and negatively charged hadron and pion multiplicities shows a flat $x$ behaviour, as expected in LO pQCD. For charged hadrons this sum is in agreement with EMC results obtained at comparable kinematics, whereas some inconsistency is observed when comparing to HERMES pion data that were taken at different kinematics. The ratio of the $z$-integrated positive and negative hadron and pion multiplicities as a function of $x$ nicely confirms the previous measurements from HERMES and EMC.

The measured charged pion multiplicities were used for a LO extraction of the favoured and unfavoured pion FFs. While both FFs are significantly different from those obtained in the HKNS fit to only the electron-positron annihilation data, they are in good agreement with those obtained in recent NLO fits that also include a preliminary release of the present data.

The sum of $K^{+}$and $K^{-}$multiplicities integrated over $z$ shows a flat distribution in $x$, with values significantly higher than those measured by HERMES at lower energy. By covering lower $x$ values, the multiplicity sum data will significantly improve the constraint on the product of the strange quark PDF and FF.

\section{References}

[1] C. Adolph et al., (COMPASS Collaboration) Physics Letters B 764, 1-10 (2017).

[2] C. Adolph et al., (COMPASS Collaboration) Physics Letters B 767, 133-141 (2017).

[3] J.C. Collins, D.E. Soper, G.F. Sterman, Nucl. Phys. B 261, 104 (1985).

[4] P. Abbon, et al., (COMPASS Collaboration) Nucl. Instrum. Methods A 577, 455 (2007).

[5] A. Airapetian, et al., (HERMES Collaboration) Phys. Rev. D 87, 074029 (2013).

[6] EMC, J. Ashman, et al., Z. Phys. C 52, 361 (1991).

[7] R. Asaturyan, et al., (JLab E00-108) Phys. Rev. C 85, 015202 (2012).

[8] A. Airapetian, et al., (HERMES Collaboration) Phys. Rev. D 89, 097101 (2014).

[9] E. Leader, A.V. Sidorov, D.B. Stamenov, Phys. Rev. D 93, 074026 (2016).

[10] M. Stolarski, Phys. Rev. D 92, 098101 (2015).

[11] E. Aschenauer, et al., Phys. Rev. D 92, 098102 (2015).

[12] A. Airapetian, et al., (HERMES Collaboration) Phys. Rev. D 87, 074029 (2013). 